Гимбатгаджиева М.М.

Кафедра нервных болезней лечебного факультета ФГБОУ ВО «Московский государственный медикостоматологический университет им. А.И. Евдокимова» Минздрава России, Москва, Россия

127473 Москва, ул. Делегатская, 20, стр. 1

\title{
Криптогенная (вероятно симптоматическая) эпилепсия
}

Рассматривается классификация эпилепсии и эпилептических синдромов. Обсуждаются место криптогенной (вероятно симптоматической) эпилепсии в ряду эпилептических синдромов, современный подход к определению криптогенной эпилепсии, особенности криптогенной эпилепсии у взрослых.

Ключевые слова: эпилепсия; криптогенная; симптоматическая. Контакты: Марьям Магомедовна Гимбатгаджиева; gtm1983@таil.ru

Для ссылки: Гимбатгаджиева ММ. Криптогенная (вероятно симптоматическая) эпилепсия. Неврология, нейропсихиатрия, психосоматика. 2017;(спецвыпуск 1):97-100.

\section{Cryptogenic (probably symptomatic) epilepsy}

Gimbatgadzhieva M.M.

Department of Nervous System Diseases, Faculty of General Medicine, A.I. Evdokimov Moscow State University of Medicine and Dentistry, Ministry of Health of Russia, Moscow, Russia; 20 Delegatskaya St., Build, 1, Moscow 127473

The paper considers the classification of epilepsy and epileptic syndromes. It discusses the place of cryptogenic (probably symptomatic) epilepsy among the epileptic syndromes, the current approach to defining cryptogenic epilepsy, and the features of the latter in adults.

Keywords: cryptogenic (symptomatic) epilepsy.

Contact: Mariam Magomedovna Gimbatgadzhieva; gtm1983@mail.ru

For reference: Gimbatgadzhieva MM. Cryptogenic (probably symptomatic) epilepsy. Nevrologiya, Neiropsikhiatriya, Psikhosomatika = Neurology, Neuropsychiatry, Psychosomatics. 2017; (Special Issue 1):97-100.

DOI: http://dx.doi.org/10.14412/2074-2711-2017-1S-97-100

Эпилепсия является одним из наиболее распространенных заболеваний нервной системы, поражающим до $0,5 \%$ населения, сопровождается повышенным риском смерти, частым развитием депрессии, снижением когнитивных функций, работоспособности и способности к обучению. Указанные факторы обусловливают снижение качества жизни больных и нарушение их трудовой и социальной адаптации [1].

Согласно данным большинства исследований, распространенность эпилепсии в разных регионах мира составляет от 1,5 до 50 случаев на 100 тыс. населения [2], в Российской Федерации - около 2,5 случая на 100 тыс. населения [3]. От эпилепсии страдает около 50 млн человек. По данным И.В. Головановой [4], в Москве распространенность эпилепсии среди взрослого населения составляет 2,34 на 100 тыс. населения. По данным Б.М. Доронина и Х.Д. Монгуш [5], большинство (почти 90\%) взрослых пациентов с эпилепсией имеют инвалидность и только треть пациентов продолжают работать. Средний возраст дебюта эпилепсии у взрослых пациентов - 23 года, т. е. заболевание поражает молодую, социально активную часть общества. Эти данные подчеркивают высокую медико-социальную и экономическую значимость проблемы эпилепсии во взрослом возрасте. Основной причиной эпилепсии во взрослом возрасте, по данным Б.М. Доронина и Х.Д. Монгуш [5], является черепно-мозговая травма, менее частые причины цереброваскулярные заболевания, последствия нейроинфекций, перинатальная патология, опухоли головного мозга, нейроцистицеркоз, хронический алкоголизм. Важно отметить, что этиология заболевания ими была определена только в $71,7 \%$ случаев, в то время как примерно у трети пациентов причина заболевания установлена не была.

В последние годы достигнуты значительные успехи в области изучения фундаментальных механизмов эпилепто- генеза, генетических и молекулярных основ развития заболевания. Предложены новые методы диагностики, включая современные нейровизуализационные методики и нейрофизиологические методы с компьютерной обработкой. Значительным достижением современной эпилептологии является внедрение в клиническую практику новых противоэпилептических препаратов, что позволяет увеличить эффективность и безопасность терапии, а также новых подходов к хирургическому лечению заболевания. В то же время многие вопросы практической эпилептологии окончательно не изучены, что выражается, в частности, в значительной доле пациентов с криптогенной формой заболевания. Это отражает отсутствие на современном этапе возможности отнести указанные случаи к вариантам заболевания с установленной этиологией [6].

Согласно определению Международной противоэпилептической лиги (ILAE) 2005 г., эпилептический приступ транзиторное (преходящее) появление признаков или симптомов, обусловленное чрезмерной аномальной синхронной нейрональной активностью, а эпилепсия - заболевание головного мозга, характеризующееся стойкой предрасположенностью к генерации эпилептических приступов с развитием нейробиологических, когнитивных, социальных и психических последствий.

Традиционно считалось, что для постановки диагноза эпилепсии необходимо наличие как минимум двух неспровоцированных эпилептических приступов [7]. Согласно определению от 2014 г. эпилепсию можно диагностировать, когда состояние отвечает одному из следующих условий: по крайней мере два неспровоцированных эпилептических приступа, наблюдаемых с интервалом >24 ч; один неспровоцированный эпилептический приступ и вероятность следующих приступов, аналогичных общему риску рецидива после двух неспровоцированных приступов (>60\%); нали- 
чие достаточной информации для постановки диагноза специфического эпилептического синдрома.

Данное определение от 2014 г. признает, что эпилепсия необязательно продолжается всю жизнь и диагноз эпилепсии можно снять у лиц, имевших возрастной эпилептический синдром, но прошедших установленный возраст, и у людей, у которых приступы не наблюдались в течение последних 10 лет и которые не принимали в последние 5 лет противоэпилептические препараты $[8,9]$.

В течение длительного времени в клинической эпилептологии используется классификация эпилепсии и эпилептических синдромов в зависимости от вида приступов (генерализованные и фокальные или парциальные) и этиологии. Этиологическая классификация приступов предполагает их разделение на симптоматические, идиопатические и криптогенные. Последнее разделение, предложенное в 1989 г., требовало пересмотра в связи с открытием новых форм заболевания и внедрением в клиническую и исследовательскую практику новых методов диагностики. В то же время до настоящего времени классификация 1989 г. остается основой для практической деятельности врачей во всем мире. Идиопатическая эпилепсия - это эпилепсия, при которой отсутствуют заболевания или структурные повреждения головного мозга, которые могли бы служить причиной развития эпилептических приступов; в этом случае эпилепсия является первичным заболеванием. В настоящее время считается, что в основе развития большинства форм идиопатической эпилепсии лежат генетические факторы. При симптоматической эпилепсии эпилептический синдром возникает в результате известных и верифицированных морфологических нарушений, т. е. приступы возникают вторично. Криптогенная эпилепсия занимает промежуточное положение между симптоматической и идиопатической эпилепсией. В этих случаях нет однозначных доказательств симптоматической природы заболевания при отсутствии критериев соответствия идиопатическим формам заболевания. При криптогенной эпилепсии клиническая и электроэнцефалографическая (ЭЭГ) картина заболевания обычно соответствует одной из локально обусловленных форм заболевания при отсутствии в анамнезе заболеваний или травм головного мозга и изменений при проведении нейровизуализации [6].

В 2001 г. ILAE был предложен новый подход к классификации эпилепсии, основанный на прогностической, терапевтической и диагностической значимости приступа. Согласно данному подходу (алгоритму), выделяют 5 уровней прогностической классификационной схемы. Следует отметить, что в указанной классификации предлагалось заменить термин «криптогенная эпилепсия» на более точный «вероятно симптоматическая эпилепсия». В группу симптоматической / вероятно симптоматической эпилепсии были включены следующие синдромы: лимбические эпилепсии (мезиальные височные эпилепсии с гиппокампальным склерозом, мезиальная височная эпилепсия специфической этиологии, другие формы), неокортикальные эпилепсии (энцефалит Расмуссена, гемиконвульсивно-гемиплегический синдром, мигрирующие парциальные приступы раннего младенчества, другие формы определенной локализации). В 2010 г. в докладе комиссии ILAE вновь предложено вернуться к изменению классификации эпилепсии по этиологии в виде отказа от использования термина «криптогенная эпилепсия» и замене его на термин «эпилепсия, причины которой неизвестны» [10].

Возвращаясь к изменению классификации эпилепсии, В.А. Карлов и О.С. Иноземцева [11] отмечают, что отечественная эпилептическая школа никогда не поддерживала концепцию выделения криптогенной формы как отдельного варианта заболевания, исходя из представлений о единстве эпилепсии как заболевания, развитие которого обусловлено сложным взаимодействием факторов окружающей среды и генетических (эндогенных) факторов.

До настоящего времени нет четкой определенности относительно нозологической самостоятельности криптогенной формы эпилепсии. Попытки изменить терминологию, заменив термин «криптогенная эпилепсия» на «вероятно симптоматическую эпилепсию» (2001) или «эпилепсию, причина которой еще неизвестна» (2010), отражают скорее отсутствие удовлетворительного объяснения причин развития данной формы заболевания и единого представления о ее этиологии. В то же время необходимо отметить, что криптогенная эпилепсия является наиболее частым вариантом заболевания у взрослых. Так, в крупных популяционных исследованиях взрослого населения с эпилепсией показано, что до $80 \%$ случаев заболевания связаны с локально обусловленными формами, в структуре которых доминируют именно криптогенные формы, а на долю идиопатических случаев приходится не более $10-12 \%$ [3, 12].

По мнению В.А. Карлова и соавт. [13], к криптогенной эпилепсии относятся случаи заболевания, когда оба фактора (генетический и органический) выражены мягко, но в совокупности обусловливают развитие заболевания. Таким образом, ведущее значение в изучении этиологии криптогенных форм заболевания могут иметь как генетические исследования, направленные на поиск генов-кандидатов с неполной пенетрантностью, которые могут увеличивать предрасположенность к развитию заболевания, так и дальнейшее изучение «мягких» структурных изменений.

Результаты исследований с применением современных методик анализа ЭЭГ демонстрируют, что при криптогенной эпилепсии у взрослых регистрируется патологические острые волны преимущественно по левым переднецентральным отведениям, а также комплексы острая-медленная волна и спайк-волна по правым лобным и центральным отведениям. Таким образом, ЭЭГ-картина криптогенной эпилепсии у взрослых позволяет говорить о некоторой очаговости патологического процесса [14].

В настоящее время для уточнения генеза криптогенной эпилепсии активно используются современные методы нейровизуализации, такие как магнитно-резонансная (МР) спектроскопия, диффузионно-тензорная трактография, МР-морфометрия, функциональная МР-томография (МРТ) и др. Использование методов нейровизуализации позволяет выявлять структурные и функциональные изменения в разных регионах мозга, что может использоваться как для уточнения патофизиологических механизмов эпилептогенеза в случаях криптогенной эпилепсии, так и для разработки диагностических и прогностических маркеров [15-17].

МР-спектроскопия является неинвазивным методом определения содержания ряда метаболитов и нейромедиаторов, таких N-ацетиласпартат (NAA), холин, мио-инозитол, глутамат и гамма-аминомасляная кислота, в регионе интереса. В исследованиях с применением этого метода показано, что для эпилептических очагов характерно уменышение содержания NAA с одновременным увеличением содержания холина или креатина, что выражается в изменении отношения NAA/холин+креатин [18-20]. В настоящее время считается, что данный индекс является одним из наиболее чувствительных для выявления эпилептических очагов в височной доле [21]. Важно отметить, что у пациентов с криптогенной формой эпилепсии анализ данного отношения позволяет идентифицировать эпилептический очаг в $87 \%$ случаев, а его локализация, определенная с помощью данного метода совпадает с клиническими данными (феноменология приступов) и результатами ЭЭГ относительно локализации фокуса эпилептической активности [22-24]. По данным М.Ү. Ни и соавт. [25], у пациентов с височ- 
ной эпилепсией и отсутствием изменений на МРТ выявляется статистически значимое уменьшение пика NAA и отношения NAA/холин+креатин на стороне, соответствующей локализации приступов. Предполагается, что в основе снижения концентрации NAA и увеличения концентрации холина и креатина в эпилептическом очаге лежат снижение нейроаксональной плотности и уменьшение количества активных функционирующих нейронов, распад мембран нервных клеток и реактивный астроглиоз [20]. Послеоперационное гистопатологическое обследование показывает, что участкам со снижением пика NAA и гипоперфузией по данным позитронно-эмиссионной томографии (ПЭТ) наиболее часто соответствуют участки фокальной кортикальной дисплазии [26].

Диффузионно-тензорная МРТ (ДТ-МРТ) является неинвазивным методом нейровизуализации, предназначенным для выявления тонких изменений белого вещества проводников ЦНС. Предполагается, что изменения аксонов и миелиновой оболочки проводящих структур приводят к нарушению диффузии и анизотропии в очагах поражения. Увеличение диффузии и уменьшение анизотропии выявляется у всех больных с различимыми при выполнении стандартной нейровизуализации очагами. В то же время у большинства больных изменения анизотропии и диффузии выявляются также в местах, расположенных вокруг эпилептического очага. Кроме того, у $80 \%$ больных с криптогенной эпилепсией могут быть выявлены увеличение диффузии и уменьшение анизотропии, что указывает на наличие скрытых очагов структурного повреждения [27-29].

С.Н. Базилевич [30] при обследовании 80 пациентов в возрасте до 40 лет выделил 4 группы больных криптогенной эпилепсией с отсутствием изменений при выполнении МРТ в стандартных режимах в зависимости от результатов использования дополнительных методов нейровизуализации:

- группа пациентов без изменений (29\%; группа 1);

- группа пациентов с локальными, «мягкими» и обратимыми изменениями (50\%; группа 2);

- группа пациентов с локальными выраженными и прогрессирующими изменениями (14\%; группа 3);

• группа пациентов с диффузными и многоочаговыми изменениями (7\%; группа 4).

Таким образом, использование дополнительных методов нейровизуализации позволяет выявить функциональные изменения у подавляющего числа больных (71\%). Автором было показано, что для каждой группы больных характерны определенные клинические и ЭЭГ-особенности. Так, для пациентов первой группы характерно наличие вторично-генерализованных эпилептических приступов или простых и сложных парциальных приступов при нормальной в большинстве случаев ЭЭГ в межприступный период. Для пациентов второй группы характерно наличие частых вторично-генерализованных простых парциальных приступов с вегетативными проявлениями или нарушением психических функций, при этом на ЭЭГ в $85 \%$ случаев выявляется эпилептиформная активность (в 100\% случаев - при выполнении функциональных проб). Для пациентов третьей группы характерны частые сложные парциальные или вторично-генерализованные приступы. Кроме того, в клинической картине при данном варианте эпилепсии отмечено наличие прогрессирующих когнитивных нарушений, тревожно-депрессивных расстройств. ЭЭГ-картина характеризуется наличием грубых изменений в лобных отделах, медиобазальных отделах височной доли, формированием зеркальных очагов. У пациентов четвертой группы были выявлены разнообразные по характеру эпилептические приступы с нормальной или слегка дезорганизованной картиной при проведении ЭЭГ, наличием нескольких независимых эпилептогенных очагов. Важной особенностью данной группы пациентов является фармакорези- стентность, несмотря на политерапию и частые изменения схемы приема противоэпилептических препаратов [30].

Одним из перспективных методов оценки функциональных нарушений отдельных регионов головного мозга у пациентов с криптогенной эпилепсией в межприступный период является ПЭТ с 18F-фтордезоксиглюкозой, позволяющая выявлять локальные участки гипометаболизма, соответствующие, предположительно, локализации эпилептических очагов. Так, например, у пациентов с височной эпилепсией и без структурных изменений на МРТ ПЭТ выявляет участки гипометаболизма в $90 \%$ случаев [31-33]. Примечательно, что, по данным одного из исследований, именно выявление зон гипометаболизма является наиболее чувствительным методом определения локализации латерализации и размеров эпилептического очага, по сравнению с другими методами (оценка толщины коры и индекс фракционной анизотропии по данным диффузионно-тензорной трактографии) [34].

Еще одним высокоинформативным методом функциональной нейровизуализации для выявления эпилептических очагов при криптогенной эпилепсии является однофотонная эмиссионная компьютерная томография (ОФЭКТ). При внутривенном введении радиофармпрепарата до начала приступа у пациентов с криптогенной эпилепсией очаг гиперперфузии может быть выявлен в 90-99\% случаев, при введении после начала приступа - в 70-75\% случаев, в межприступный период - в $40 \%$ случаев. Крайне важно, что очаги гиперперфузии при криптогенной эпилепсии с помощью ОФЭКТ могут быть выявлены не только при височной, но и при лобной, теменной и затылочной локализации патологического процесса. Предполагается, что выявление очагов гиперперфузии с помощью ОФЭКТ во время и сразу после приступа отражает увеличение нейрональной активности, что приводит к локальному увеличению перфузии [17, 35].

В исследовании, проведенном M.C. Vlooswijk и соавт. [36], показано, что у взрослых пациентов с криптогенной эпилепсией, по данным функциональной МРТ, наблюдается уменьшение функциональных связей между регионами префронтальной коры, нижней лобной извилиной, что коррелирует со снижением оперативной памяти по данным выполнения нейропсихологического тестирования. Важно отметить, что объем гиппокампа при криптогенной эпилепсии статистически значимо не отличался от нормы. Таким образом, в части случаев при криптогенной эпилепсии могут выявляться функциональные изменения, отражающие нарушения взаимодействия различных регионов головного мозга.

В.А. Карлов никогда не поддерживал предложение ILAE считать криптогенную эпилепсию вероятно симптоматической. В его монографии от 1990 г. [37] эпилепсия рассматривалась как единая болезнь - следствие взаимодействия предрасположенности и экзогенных факторов, признавалось существование наследственной эпилепсии как формы заболевания, обусловленной необычно повышенной предрасположенностью к эпилепсии. При этом автором отмечается возможная наследственная передача посредством трансмиссии доминантного аутосомного гена с нерегулярной пенетрантностью. Влияние такого гена зависит от влияния окружающей среды. В связи с этим нельзя не вспомнить выдающегося отечественного невролога С.Н. Давиденкова, который рассматривал популяцию больных эпилепсией как континуум, в ряду которого слева направо убывает роль генетического фактора и возрастает роль органического поражения головного мозга. И наоборот, справа налево возрастает роль генетического фактора и убывает в соответствии с этим роль органического поражения мозга. С.Н. Давиденков рассматривал крайние составляющие континуума как первично генерализованную (генуинную) и очаговую (симптоматическую) эпилепсию. 
Криптогенная эпилепсия является наиболее частым вариантом эпилепсии у взрослого населения. До настоящего времени отсутствуют общепринятые представления об этиологии и патогенезе данной формы заболевания. Современные исследования, в том числе с применением новейших методов структурной и функциональной нейровизуализа- ции, демонстрируют, что в основе криптогенной эпилепсии могут лежать полиморфные изменения, что, вероятно, отражается в многообразии клинических и ЭЭГ-проявлений и определяет прогноз и ответ на терапию. Дальнейшее изучение криптогенной эпилепсии у взрослых может играть важную роль в совершенствовании ее диагностики и лечения.

\section{Л И Т E P A T V P A}

1. Tellez-Zenteno J, Ronquillo F, Wiebe S. Sudden unexpected death in epilepsy: evidencebased analysis of incidence and risk factors. Epilepsy Res.

2005;65:101-15. doi: 10.1016/j.eplepsyres.2005.05.004 2. Helmers SL, Thurman DJ, Durgin TL, et al.

Descriptive epidemiology of epilepsy in the U.S. population: A different approach. Epilepsia. 2015;56(6):9428. doi: 10.1111/epi.13001

3. Гехт АБ, Мильчакова ЛЕ и др. Исследование эпидемиологии эпилепсии в РФ. Журнал неврологии и психиатрии им. С.С. Корсакова. 2006;106:37 [Guekht A, Milchakova L, et al. The research of epidemiology of epilepsy in the Russian Federation. Zhurnal Nevrologii i Psikhiatrii im. S.S. Korsakova $=$ SS Korsakov Journal of Neurology and Psychiatry. 2006;106:3-7 (In Russ.)].

4. Голованова ИВ. Клинико-эпидемиологическое исследование эпилепсии в Москве: Автореф. дис. . канд. мед. наук. Москва; 2003 [Golovanova IV. Kliniko-epidemiologicheskoe issledovanie epilepsii v Moskve: Avtoref. dis. ... kand. med. nauk [Clinical and epidemiological study of epilepsy in Moscow: Author's abstract. Dis. ... cand. Med. Sci.]. Moscow; 2003] 5. Доронин БМ, Монгуш ХД. Эпидемиология эпилепсии в Республике Тыва. Журнал неврологии и психиатрии им. С.С. Корсакова. 2011;111(3) 64-6 [Doronin BM, Mongush KhD. Epidemiology of epilepsy in the Republic of Tuva. Zhurnal Nevrologii $i$ Psikhiatrii im. S.S. Korsakova $=$ SS Korsakov Journal of Neurology and Psychiatry. 2011; 111(3):64-66 (In Russ.)]. 6. Карлов ВА. Эпилепсия у мужчин и женщин взрослых и детей. Москва: Медицина; 2010. 720 с. [Karlov WA. Epilepsy at children and adult, women and men. Moscow: Meditsina; 2010. 720 p.]. 7. Fisher RS, van Emde Boas W, Blume W, et al. Epileptic seizures and epilepsy: definitions proposed by the International League Against Epilepsy (ILAE) and the International Bureau for Epilepsy (IBE). Epilepsia. 2005;46:470-2. doi: 10.1111/j.00139580.2005.66104.x

8. Fisher RS, Acevedo C, Arzimanoglou A et al. ILAE official report: a practical clinical definition of epilepsy. Epilepsia. 2014;55(4):475-82. doi: 10.1111/epi.12550 9. Fisher RS. Redefining epilepsy. Curr Opin Neurol. 2015;28(2):130-5. doi: 10.1097/WCO.0000000 000000174

10. Berg AT, Berkovic SF, Brodie MJ, et al. Revised terminology and concepts for organization of seizures and epilepsies: Report of the ILAE Commission on Classification and Terminology, 2005-2009. Epilepsia. 2010;51(4):676-85. doi: 10.1111/j.1528-1167. 2010.02522.x

11. Карлов ВА, Иноземцева ОС. Современный подход к определению криптогенной формы эпилепсии. Журнал неврологии и психиатрии им. C.C. Корсакова. 2013;113(4-2):20-1 [Karlov VA, Inozemtseva OS. Modern approach to the definition of cryptogenic forms of epilepsy. Zhurnal Nevrologii $i$

Psikhiatrii im. S.S. Korsakova $=$ SS Korsakov Journal of Neurology and Psychiatry. 2013;113(4):20-1 (In Russ.)]. 12. Хаузер ВА. Описательная эпидемиология эпилепсии. Москва; 2011. С. 9-41 [Hauser WA. The descriptive epidemiology of epilepsy. Moscow; 2011. P. 9-41].

13. Карлов ВА, Ожерельева ЮВ, Коваленко ГА. Криптогенные эпилепсии - фенокопии идиопа тических эпилепсий? Журнал неврологии и психиатрии им. С.С. Корсакова. 2011;111(2):14-7 [Karlov VA, Ozhereleva YV, Kovalenko GA. Cryptogenic epilepsy - modification of idiopathic epilepsy? Zhurnal Nevrologii i Psikhiatrii im. S.S. Korsakova $=$ SS Korsakov Journal of Neurology and Psychiatry. 2011;111(2):14-7 (In Russ.)].

14. Егорова НВ. Фармакоэпидемиология, аспекты формирования резистентности к лечению у пациентов с различными формами эпилепсии в Смоленской области: Автореф. дисс. .... канд. мед. наук. Москва; 2009. 18 с. [Egorova NV.

Farmakoepidemiologiya, aspekty formirovaniya rezistentnosti $k$ lecheniyu u patsientov s razlichnymi formami epilepsii v Smolenskoi oblasti: Avtoref. diss. ... kand. med. nauk [Pharmacoepidemiology, aspects of the formation of resistance to treatment in patients with different forms of epilepsy in Smolensk region: Author. Diss. ... Cand. Med. Sci]. Moscow; 2009. 18 p.] 15. Bernasconi A, Bernasconi N, Bernhardt BC, et al. Advances in MRI for «cryptogenic» epilepsies. Nat Rev Neurol. 2011;7(2):99-108. doi: 10.1038/nrneurol. 2010.199

16. Jansen JF, van der Kruijs SJ, Vlooswijk MC, et al. Quantitative MR and cognitive impairment in cryptogenic localisation-related epilepsy. Epileptic Disord. 2014;16(3):318-27.

17. Pardoe H, Kuzniecky R. Advanced Imaging Techniques in the Diagnosis of Nonlesional Epilepsy: MRI, MRS, PET, and SPECT. Epilepsy Curr. 2014;14(3):121-4 doi: 10.5698/1535-7597-14.3.121 18. Hugg JW, Laxer KD, Matson GB, et al. Neuron loss localizes human temporal lobe epilepsy by in vivo proton magnetic resonance spectroscopic imaging. An Neurol. 1993;34:788-94. doi: 10.1002/ana.410340606 19. Bates TE, Strangward M, Keelan J, et al. Inhibition of $\mathrm{N}$-acetylaspartate production: implications for 1H MRS studies in vivo. Neuroreport 1996;7:1397-400. doi: 10.1097/00001756-19960531000014

20. Pan JW, Kuzniecky RI. Utility of magnetic resonance spectroscopic imaging for human epilepsy. Quant Imaging Med Surg. 2015;5(2):313-22. 21. Bernasconi A, Tasch E, Cendes F, et al. Proton magnetic resonance spectroscopic imaging suggests progressive neuronal damage in human temporal lobe epilepsy. Prog Brain Res. 2002;135:297-304. doi: 10.1016/S0079-6123(02)35027-1

22. Cendes F, Andermann F, Preul MC,

Arnold DL. Lateralization of temporal lobe epilepsy based on regional metabolic abnormalities in proton magnetic resonance spectroscopic images. Ann Neurol. 1994;35:211-6. doi: 10.1002/ana.410350213

23. Cendes F, Caramanos Z, Andermann F, et al. Proton magnetic resonance spectroscopic imaging and magnetic resonance imaging volumetry in the lateralization of temporal lobe epilepsy: a series of 100 patients. Ann Neurol. 1997;42:737-46. doi: 10.1002/ana.410420510
24. Pan JW, Spencer DD, Kuzniecky R, et al. Metabolic networks in epilepsy by MR spectroscopic imaging. Acta Neurol Scand. 2012;126:411-20. doi: 10.1111/j.1600-0404.2012.01665.x 25. Xu MY, Ergene E, Zagardo M, et al. Proton MR Spectroscopy in Patients with Structural MRINegative Temporal Lobe Epilepsy. J Neuroimaging 2015;25(6):1030-7. doi: 10.1111/jon. 12263

26. Krsek P, Hajek M, Dezortova M, et al. (1)H MR spectroscopic imaging in patients with MRI-negative extratemporal epilepsy: correlation with ictal onset zone and histopathology. Eur Radiol. 2007;17(8):212635. doi: 10.1007/s00330-007-0594-1

27. Duning T, Kellinghaus C, Mohammadi S, et al. Individual white matter fractional anisotropy analysis on patients with MRI negative partial epilepsy. J Neurol Neurosurg Psychiatry. 2010;81(2):136-9. doi: $10.1136 /$ jnnp.2008.160820

28. Wang ZI, Alexopoulos AV, Jones SE, et al. The pathology of magnetic-resonance-imaging-negative epilepsy. Mod Pathol. 2013;26(8):1051-8.

doi: $10.1038 /$ modpathol.2013.52

29. Peng SJ, Harnod T, Tsai JZ, et al. Evaluation of subcortical grey matter abnormalities in patients with MRI-negative cortical epilepsy determined through structural and tensor magnetic resonance imaging. BMC Neurol. 2014;14:104. doi: 10.1186/1471-237714-104

30. Базилевич СН. Криптогенная эпилепсия у взрослых: скрытые проблемы структурного благополучия. Журнал неврологии и психиатрии им. C.C. Корсакова. 2013;113(4):10-9 [Bazilevich SN Cryptogenic epilepsy in adults: hidden problems of structural well-being. Zhurnal Nevrologii i Psikhiatrii im. S.S. Korsakova $=$ SS Korsakov Journal of Neurology and Psychiatry. 2013;113(4):10-9 (In Russ.)]. 31. Schöll M, Damian A, Engler H. Fluorodeoxyglucose PET in Neurology and Psychiatry. PET Clin. 2014;9(4):371-90. doi: 10.1016/j.cpet.2014.07.005 32. Feng R, Hu J, Pan L, et al. Surgical treatment of MRI-negative temporal lobe epilepsy based on PET: a retrospective cohort study. Stereotact Funct Neurosurg. 2014;92(6):354-9. doi: 10.1159/000365575

33. Burneo JG, Poon R, Kellett S, Snead OC. The Utility of Positron Emission Tomography in Epilepsy. Can J Neurol Sci. 2015;6:1-12. doi: 10.1017/cjn.2015.279 34. Pustina D, Avants B, Sperling M, et al. Predicting the laterality of temporal lobe epilepsy from PET, MRI, and DTI: A multimodal study. Neuroimage Clin. 2015;9:20-31. doi: 10.1016/j.nicl.2015.07.010 35. Joo EY, Seo DW, Hong SC, et al. Functional neuroimaging findings in patients with lateral and mesiolateral temporal lobe epilepsy; FDG-PET and ictal SPECT studies. J Neurol. 2015;262(5):1120-9. doi: 10.1007/s00415-014-7625-Z 36. Vlooswijk MC, Jansen JF, Jeukens CR, et al. Memory processes and prefrontal network dysfunction in cryptogenic epilepsy. Epilepsia. 2011;52(8): 1467-75. doi 10.1111/j.1528-1167.2011.03108.x 37. Карлов ВА. Эпилепсия. Москва: Медицина; 1990. 336 c. [Karlov VA. Epilepsiya [Epilepsy]. Moscow: Meditsina; 1990. 336 p.].

Поступила 16.04.2017

Исследование не имело спонсорской поддержки. Автор несет полную ответственность за предоставление окончательной версии рукописи в печать. Окончательная версия рукописи была одобрена автором. 